\title{
El papel del conocimiento escolar universitario
}

\section{The role of the university school knowledge}

\author{
Análida Elizabeth Pinilla • Bogotá, D.C. (Colombia) \\ TiBURCIO MoReno • MÉXICO D.F. (MÉXICO)
}

\section{Resumen}

Se categoriza el conocimiento cotidiano y científico que conforman el conocimiento escolar universitario o académico; se sitúa al profesor universitario como el actor principal que genera, gestiona, integra, a su vez, transforma conocimientos frente al estudiante; se realza la transposición didáctica compleja en ciencias de la salud, dado que la acción del especialista/docente es para proponer soluciones a la diversidad de problemas de cada paciente.

Luego se plantea una reforma académica con un currículo flexible no enciclopedista, que facilite la elaboración del conocimiento por parte de los alumnos. A continuación se argumenta acerca de qué enseñar de acuerdo con la epidemiología clínica, pero avanzando más allá del conocimiento específico para proponer el desarrollo de diversas capacidades o competencias específicas, transversales y sociales para la vida; por qué enseñar, según la postura epistemológica y el conocimiento profesional del profesor y la postura que va desarrollando un estudiante.

Así mismo se discute sobre la ontología del conocimiento, ciencia e investigación y cómo un estudiante universitario construye de forma permanente su propio conocimiento en interacción con su medio social y cultural.

Finalmente se invita al trabajo universitario con metodologías activas, centradas en el estudiante con un profesor tutor, que apoya el desarrollo de competencias (que integran conocimientos, habilidades y actitudes) con diverso grado de complejidad y profundidad según el nivel de formación. Para concluir, se invita a los profesores universitarios a responder con sus funciones de docencia, investigación y extensión con el compromiso de apoyar estudiantes de pregrado y posgrado. (Acta Med Colomb 2015; 40: 241-245).

Palabras clave: maestro, estudiante, conocimiento, docencia, investigación, extensión.

\begin{abstract}
Daily and scientific knowledge that make up the university or academic school knowledge is categorized; the university professor is situated as the main actor who creates, manages, integrates, and in turn transforms knowledge in front of the student; the complex didactic transposition in health sciences is enhanced, since the action of the specialist / teacher is to propose solutions to the diverse problems of each patient.

Next, an academic reform with a flexible non encyclopedic curriculum facilitating the elaboration of knowledge by students is proposed. Then, the argument about what to teach according to clinical epidemiology, but moving beyond the specific knowledge to propose the development of different capacities or specific, transversal and social life skills; why teach, according to the epistemological position and professional knowledge of the teacher and the position that a student is developing.

Likewise, the ontology of knowledge, science and research and the way a college student permanently builds his own knowledge in interaction with his social and cultural environment, is discussed.

Finally, university work with active methodologies centered in the student with a tutor, who supports skills development (integrating knowledge, skills and attitudes) with varying degrees of complexity and depth according to the level of training, is encouraged. To conclude, the university professors are invited to respond with their teaching, research and extension with the commitment to support undergraduate and graduate students. (Acta Med Colomb 2015; 40: 241-245).

Keywords: teacher, student, knowledge, teaching, research, extension.
\end{abstract}

Dra. Análida Elizabeth Pinilla Roa: Internista. Formación en Nutrición y Diabetes. PhD. en Educación. MSc. en Educación con Énfasis en Docencia Universitaria. Coordinadora Grupo de Apoyo Pedagógico y Formación Docente. Profesora Asociada Departamento de Medicina Interna, Facultad de Medicina, Universidad Nacional de Colombia. Bogotá, D.C. (Colombia); Dr. Tiburcio Moreno Olivos: Doctor en Pedagogía. Profesor Investigador Titular. Miembro del Grupo Institucional de Investigación Educativa, División de Ciencias de la Comunicación y Diseño, Departamento de Tecnologías de la Información. Universidad Autónoma Metropolitana-Cuajimalpa, México. Correspondencia. Dra. Análida Elizabeth Pinilla Roa. Bogotá, D.C. (Colombia).

E-mail: aepinillar@unal.edu.co

E-mail: tmoreno@correo.cua.uam.mx Recibido: 2/IV/2015 Aceptado: 28/V/2015 


\section{Introducción}

¿Cómo trascender del conocimiento cotidiano hacia el conocimiento científico? Esta mediación se da en el conocimiento escolar elaborado en la universidad, ¿qué es el conocimiento escolar?, ¿cuál es la razón de ser del conocimiento escolar universitario? Para responder, es necesario reflexionar sobre ¿qué se enseña?, ¿cómo se enseña?, ¿cuándo se enseña?, ¿para qué se enseña? Y, en esta misma secuencia de análisis, pensar en los procesos de enseñanza, aprendizaje y evaluación en los que el sujeto activo es el estudiante de pregrado o de posgrado. El profesor universitario se verá enfrentado a diversos grupos, pero en esencia a cada persona en la posición de estudiante con su historia de vida particular y con su propio proyecto de vida.

Para comenzar,el profesor cuando integra el conocimiento científico con el conocimiento cotidiano crea conocimiento escolar universitario y, a la vez, lo transforma o adecua para hacerlo enseñable, es el conocimiento que se discute en el aula de clase, en esa interacción del maestro con su(s) alumno(s); en palabras de Chevallard (1998) el maestro hace la transposición didáctica para hacer el conocimiento comprensible con el fin de facilitar el aprendizaje y la apropiación de conceptos (1). En el caso de las ciencias de la salud, en particular en el área de práctica clínica y quirúrgica, este proceso se hace más complejo porque al trabajar frente al paciente, se tienen que integrar multiplicidad de conocimientos de las ciencias básicas para dar atención al paciente y poder resolver el o los problemas por los que consulta.

Para continuar, el alumno se acerca al mundo de una disciplina o profesión. En primer lugar, desde su cotidianidad y su cultura. En segundo lugar, en el mundo escolar o académico. Por este camino busca apropiarse del conocimiento científico mediante el cual debe capacitarse para, como afirma García, "gestionar adecuadamente su vida y su entorno" (p. 10) (2).

Estos participantes, profesor y estudiante deben trabajar alrededor de diferentes tipos de conocimiento. Una nueva mirada es acabar con la separación o ruptura entre los diferentes tipos de conocimiento, es ubicar el papel de la universidad, es decir, del profesor universitario, como mediador de los diferentes tipos de conocimiento y gestor del conocimiento. En palabras de García (1998, p. 10) es contemplar:

[...] la posibilidad de una cultura escolar que ayude a superar la dicotomía entre el pensamiento cotidiano simple -mayoritario en la población- y un pensamiento científico especializado, más complejo y minoritario, o no considerar la construcción, en el ámbito escolar, de formas de conocimiento generales que permitan a los ciudadanos una gestión más adecuada de sus problemas cotidianos (2).

\section{Qué implica una reforma académica con un currículo flexible no enciclopedista}

Las condiciones de la enseñanza a comienzo del nuevo siglo han cambiado, como han puesto de manifiesto diversos estudios, lo que exige, a la par, una formación acorde con estas exigencias que no son las del siglo pasado. Esto significa que la enseñanza universitaria no puede impartirse como tradicionalmente se ha hecho, porque ya no se trata que los alumnos acumulen una cantidad ingente de conocimientos, datos e información, sin comprender, muchas veces, para qué les va a servir en un futuro, sino que se busca que desarrollen competencias para la vida personal y profesional. Al respecto, Moreno (2009) señala: "por eso es que las orientaciones principales en la enseñanza y el papel de los profesores se han resituado, en el sentido de proporcionar entornos de experiencias que faciliten la elaboración del conocimiento por parte de los alumnos" (p. 116) (3).

La esencia de los cambios en los planes de estudio, como parte del currículo de pregrado o posgrado en la universidad, impone que cada profesor tenga conciencia de su papel con la diversidad de funciones de docencia, extensión (proyección social) e investigación. Sin embargo, se requiere resaltar la función de la docencia universitaria en la formación de estudiantes en diferentes disciplinas y profesiones que se van a desempeñar en la sociedad, en particular en un país. Por esto Agueda y Cruz (2005), afirman la importancia que tiene "la capacidad del profesorado en orientar su misión hacia las verdaderas necesidades sociales, y de sus alumnos en particular" (p.15) (4).

\section{Qué enseñar}

Cuando un comité asesor o un profesor define un conjunto de conocimientos (temas) para un programa de pregrado o posgrado, está reflejando un punto de vista del mundo.

En este sentido, se enseña en la universidad "escuela" un conocimiento que tiene diferentes fuentes; en el caso del área de la salud, los problemas sociales y de salud cotidianos (organizados por la epidemiología clínica), el conocimiento científico comprendido desde la perspectiva de la evolución y reconceptualización de los conceptos científicos. Entonces, García (1998) propone "no se trata de acercarse a lo social desde la ciencia sino a la ciencia desde lo social... El conocimiento científico tendría por tanto, el papel de un medio más que de un fin" (p. 20) (2).

Al día de hoy, Pinilla (2015) plantea qué enseñar tiene un abanico de opciones que van desde el conocimiento específico de la carrera pasando por el desarrollo de otras capacidades o competencias (tanto específicas de la profesión como transversales y sociales para la vida), que el futuro profesional debe desarrollar, las cuales difícilmente se podrían alcanzar si se continúa con una enseñanza tradicional (5).

\section{Por qué enseñar}

Se debe traspasar la meta de la enseñanza de ciertos temas, que ojalá sean seleccionados de la realidad social y del avance histórico del conocimiento científico, pero lo central para el docente y el estudiante es comprender cuál 
es la postura epistemológica que tiene el profesor y cuál la que va desarrollando el estudiante.

Al reflexionar sobre por qué enseñar, García (1998), hace referencia al conocimiento metadisciplinar acorde con la postura epistemológica, "el conocimiento sobre la naturaleza de los conocimientos disciplinares y no disciplinares", es decir, la disciplina o profesión (p. 62), la reflexión sobre el conocimiento y su evolución, la razón de ser de una determinada pregunta de investigación. Se refiere al conocimiento que conduce al profesor a tomar una decisión en el ámbito de su conocimiento profesional como profesor (2).

\section{Ontología de los diferentes tipos de conocimiento}

Se intentará dar respuesta al cuestionamiento planteado por algunos como antagonismo entre los diferentes tipos de conocimiento. Es claro que en el mundo real existe una gama o abanico de conocimientos y plantearíamos la siguiente hipótesis: la función del profesor es integrar el conocimiento cotidiano con el científico, lo cual logra no sólo por la reflexión, sino también por los procesos de investigación, con la meta de generar conocimiento escolar autóctono y quizás llegar a incidir en la cultura.

Como lo propone García (1998), el conocimiento metadisciplinar o transdisciplinar da una visión amplia de temas de varias disciplinas que se pueden disgregar y agregar para resolver un problema. Si el docente universitario incorpora este tipo de "instrumento intelectual" (p. 72) podrá seleccionar conceptos clave o fundamentales de la asignatura que desarrolla como conocimiento académico, entendido como el que se presenta, analiza y discute; es decir, se enseña y aprende en el aula. En palabras de Taba, referenciado por García, "lo esencial en la determinación de los contenidos es la selección del conjunto de principios que constituyen el núcleo básico de la materia que se va a enseñar" (p. 73) (2).

Por eso, en la gestión de la investigación en los currículos universitarios deben contemplarse los problemas socioambientales (salud, planificación del territorio, contaminación, marginación de minorías, mejora de la calidad de vida, control demográfico, etc.) los cuales atraviesan diversas disciplinas.

\section{Conocimiento cotidiano o popular}

Se ha denominado conocimiento del sentido común; la persona lo asume como dogma, sin argumentación explicativa. Es el conocimiento "experiencial, ligado a la acción" (García, 1998) corresponde al que cada persona emplea en el día a día. Es concreto y tiene un uso inmediato para poder vivir; usualmente, pasa de generación en generación en las diversas culturas. Este se aprende sin racionalizar y sin mayor explicación; no posee estructura conceptual definida, por tanto suele estar implícito en las mentes y en las culturas, sin expresarse con palabras, sino con acciones reflejas. Este autor describe otras características de este conocimiento: natural y "poco abierto a los cambios" (1998, p. 50) (2).
Corresponde al conocimiento de los alumnos que está basado en reglas heurísticas más que en la construcción y reconstrucción de conceptos; puede contener ideas o nociones implícitas y explicitas. En otras palabras se busca la solución de un problema mediante métodos no rigurosos, como por tanteo, ensayo-error, para descubrir una solución entre varias alternativas (6). García (1998) lo resume así: "El conocimiento cotidiano tiene un carácter funcional y adaptativo, siendo un saber válido para el tratamiento de los problemas propios de la vida cotidiana" (p. 29) (2).

\section{Conocimiento científico}

Plantear el concepto de conocimiento científico relativo a la ciencia impone una revisión de la historia y la filosofía de la ciencia, pues la ciencia ha tenido una evolución. El docente universitario debe concientizarse de la importancia de su posición frente a la ciencia y de la concepción sobre ésta para definir su papel en la misión de la universidad como entidad generadora de conocimiento, de forma que pueda definir qué debe enseñar y qué necesita aprender el estudiante y él mismo. Muchas veces la enseñanza de las ciencias y la organización de las asignaturas carecen de una fundamentación y de criterios claros acerca de lo que ha sido la ciencia y lo que es actualmente; conocer y comprender por qué se ha desarrollado, cuáles fueron las condiciones históricas y sociales que la hicieron posible y, en el caso de las ciencias de la salud, cómo se han ido renovando los conceptos de fisiología, fisiopatología, etiología, prevención y tratamiento, entre otros. La no comprensión de esta dinámica conduce al manejo de un concepto dogmático, ahistórico y vago de ciencia y conocimiento científico, como si se tratara de algo definitivamente hecho y simplemente acumulado en el transcurso del tiempo. En consecuencia, el docente universitario quedaría sometido a transmitir, recitar y repetir concepciones y teorías científicas con o sin fundamentos, desarrollados por otros, con el afán de la novedad, de lucirse frente a sus discípulos o simplemente simular que ha apropiado el avance de los conceptos científicos.

En el momento actual se afirma que el conocimiento no preexiste al acto de conocer, sino que es una construcción realizada por el sujeto a partir de las teorías científicas vigentes. El hombre construye conocimiento, no lo encuentra estático ni acabado en el mundo exterior, sino que lo reelabora permanentemente y lo usa en la interacción social debatiéndolo y argumentándolo. Los conocimientos científicos van evolucionando, de igual forma el alumno va edificando nuevos conceptos, que pueden llegar a tener cierto estatus temporal, con base en el bagaje de sus concepciones o ideas previas.

Por tanto, el conocimiento científico corresponde a un conocimiento más abstracto, general, que en muchas ocasiones está alejado de las vivencias del individuo. Se va configurando por un proceso complejo de indagación, reflexión, investigación que implica la evolución y la reconstrucción conceptual compleja con una lógica interna. En consecuencia, 
es explícito, se socializa y es validado por las comunidades científicas.

Para caracterizar más el concepto de conocimiento científico, es necesario preguntarse si existen diversos tipos de ciencia y cómo ha evolucionado este concepto en sí, cómo han sido los aportes de la nueva filosofía de la ciencia según diversos autores como Kuhn, Feyeraben, Toulmin, para romper "el paradigma positivista mecanicista" (p. 35) (2).

Es necesario precisar que la investigación tradicional se entiende como la fundamentada en el método científico, que es hipotético-deductivo; así, Bonilla-Castro y Rodríguez (1995) explican "lo que implica que los científicos de cualquier disciplina están básicamente probando hipótesis, el método científico así aceptado jerarquiza, ordena y regula un proceso objetivo, precepto que debe ser satisfecho por un investigador neutral" (p. 36) (7). De otra parte, la investigación cualitativa se interesa en captar la realidad social para llegar a conceptualizar, por esto no comienza desde las teorías probadas para definir variables deductivas, es decir, no emplea el método deductivo sino el inductivo; comienza con la particularidad de aceptar una realidad o un contexto social (comportamientos, creencias, opiniones, valores), luego interpreta la información tomada de ella para permitir que emerjan las categorías inductivas que van hacia la conceptualización específica de la realidad estudiada (7).

Actualmente la división clásica entre ciencias naturales y sociales está devaluada. Al día de hoy existen ciencias interdisciplinares y metadisciplinares, como las ciencias de la salud, las ciencias de la educación y la ecología.

De igual forma, los conceptos de ciencia y de conocimiento científico se han renovado. Mediante la investigación y la interacción social los paradigmas vigentes se renuevan y transforman permanentemente. Kuhn introdujo el concepto de paradigma en la historia de la ciencia y de una ciencia que se va desarrollando a través de revoluciones cuando los paradigmas compartidos por las comunidades científicas son superados por unos nuevos con mayor poder explicativo (8). La ciencia como construcción social de un conocimiento sistemático, es la principal fuerza de producción y herramienta de todos los pueblos; por ello debe estar al servicio del hombre y no ser convertida en una mercancía más, porque pierde su fundamento.

En la universidad, el conocimiento científico es medio y es fin; medio porque es el objeto de saber al reconocer la evolución y origen de los conceptos científicos, pero fin porque el maestro y el discípulo indagan permanentemente el conocimiento disponible, lo ponen a prueba y encuentran vacíos que llevan a generar preguntas de investigación y por tanto, proyectos.

\section{Conocimiento del estudiante universitario}

El conocimiento que posee el estudiante está en proceso de construcción activa, en interacción con su medio social y cultural; no es estático, sino que como proceso cambia, se modifica y evoluciona. Ha recibido diversas denominacio- nes como conocimiento previo, Driver (1978) lo identificó como "esquemas conceptuales alternativos" o "modos de ver" de los cuales disponen los estudiantes y los ensayan en nuevas situaciones; Watts (1982) se refirió a éste como de ideas espontáneas, preconceptos, esquemas conceptuales alternativos; Vigotski (1973) como prehistoria del aprendizaje; Piaget (1971) como representaciones espontáneas; y Novack (1987) como "lo que el alumno ya sabe". Cualquiera que sea la posición adoptada por el docente, se reconoce la importancia y la significación de identificar las concepciones de los alumnos (9).

El conocimiento que posee un estudiante tiene diversas características: suele tener algún grado de conflicto con los principios y conceptos científicos. Para el estudiante sus conocimientos son coherentes y tienen un poder explicativo, por tanto es difícil cambiarlos. Pueden convertirse en obstáculo para avanzar en el aprendizaje, se expresan en términos del lenguaje cotidiano. Se ha planteado que su evolución es análoga a la que ha tenido la historia de la ciencia.

Por lo anterior, es crucial que el profesor al comenzar un tema, una asignatura o una rotación con los nuevos estudiantes, explore estas concepciones e identifique los posibles errores conceptuales para aprovecharlos en el diálogo y la presentación de los temas y conceptos.

\section{Construcción del conocimiento escolar universitario 0 académico}

Aquí surgen preguntas como ¿cuál es el conocimiento que se enseña y aprende en la universidad y a quién corresponde desarrollarlo?

En el aula universitaria confluyen diversos conocimientos para la elaboración del conocimiento escolar universitario o académico: cotidiano o popular, científico, contextual, que se debaten junto al conocimiento del estudiante y el conocimiento profesional del profesor (disciplinar-profesional, curricular, pedagógico, didáctico, práctico, en investigación, en comunicación -segunda lengua, tecnológico, epistemológico y ético) (Figura 1).

Para responder estas inquietudes, en la universidad del siglo XXI, profesor y alumno deben trabajar con metodologías centradas en el discípulo y sobre temas pensados con base en las necesidades de la sociedad y en los avances y problemas de las diferentes áreas del conocimiento. Así los egresados de pregrado y posgrado como lo expresa García 2006 "serán capaces de integrar diferentes conocimientos y gestionar sistemas complejos en cualquier caso, contemplando las responsabilidades sociales y éticas. Serán capaces de aplicar los conocimientos adquiridos en ambientes profesionales nuevos" (p. 255) (10).

El trabajo en el ámbito universitario ha sido orientado tradicionalmente hacia el conocimiento disciplinar y profesional, con metodologías enfocadas en el profesor transmisor, como en la clase magistral. Ahora se requiere una nueva postura epistemológica del maestro y el discípulo universitarios para trabajar con metodologías activas, cen- 


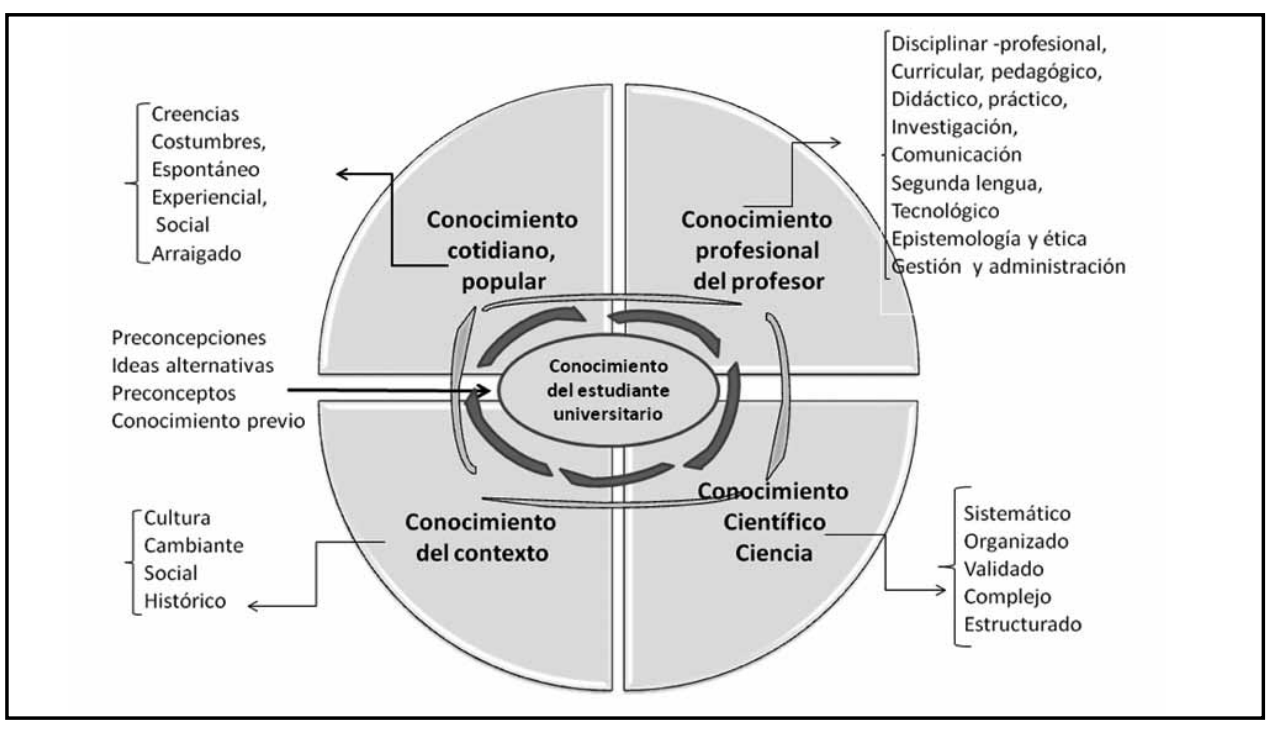

Figura 1. Emergencia y generación del conocimiento del escolar universitario. Fuente Análida E. Pinilla-Roa.

tradas en el estudiante y en las que el profesor pasa a ser el guía y tutor, de modo que el foco de aprendizaje sea no sólo la adquisición de conocimientos teóricos, sino que se amplíe hacia una diversidad de competencias que integran conocimientos, habilidades y actitudes, las cuales debe desarrollar el estudiante cada vez con más complejidad y profundidad de acuerdo con el nivel de formación universitaria y los diferentes conocimientos que aportan a la elaboración del conocimiento escolar universitario $(11,12)$.

\section{A manera de reflexiones finales}

En suma, se propone que en la universidad el equipo docente-discente trabaje integrado a diferentes profesiones y disciplinas para encontrar la continuidad e integración entre los diferentes conocimientos cotidiano, científico, contextual, académico o escolar, el del estudiante y el del profesor. Estos actores deben propiciar la renovación permanente y la complejidad progresiva del conocimiento científico. Como lo proponen Pinilla y Páramo (2011) se debe dar relevancia a la postura epistemológica del maestro universitario-investigador acerca de lo que le aporta cada tipo de conocimiento para tomar decisiones y poder responder dignamente con sus funciones de docencia, investigación y extensión en las cuales tiene el compromiso de incluir al estudiante desde el pregrado hasta el posgrado (Figura 1) (13).

Se invita a la recreación del imaginario y concepción de ciencia como aquella que está acabada por la que está en constante renovación y construcción con la participación de discípulos y docentes. A su vez, a minimizar la ruptura epistemológica entre los diferentes tipos conocimiento -cotidiano, académico y científico- los cuales aportan a la solución de problemas del mundo real y, para mejorar el bienestar de cada individuo, las comunidades y la sociedad en general. Así, se busca ubicar el papel de la universidad con sus profesores para integrar la sociedad y el mundo real con la academia y la investigación.
Se invita a los docentes universitarios a reflexionar acerca de su postura frente al conocimiento, si es que consideran que: lo transmiten, lo enseñan, apoyan su aprendizaje, lo gestionan, lo indagan, a su vez, para que analicen si son actores esenciales para la generación de proyectos de investigación. En este sentido, el maestro ha de permitir que un estudiante, de pregrado o posgrado, se apropie e integre el conocimiento en sus competencias profesionales para crear y recrear soluciones inéditas, oportunas, particulares y pertinentes a los problemas que surjan, día a día, como ciudadanos y profesionales en su desempeño (5).

\section{Referencias}

1. Chevallard Y. La transposición didáctica. Del saber sabio al saber enseñado. Buenos Aires: Aique; 1998.

2. García E. Hacia una teoría alternativa sobre los contenidos escolares. Sevilla: Díada; 1998.

3. Moreno T. La enseñanza universitaria: una tarea compleja. Revista de Educación Superior, ANUIES, México 2009; XXXVIII (3): 115-38.

4. Agueda B, Cruz A. Nuevas claves para la docencia universitaria en el EEES Madrid: Narcea S.A. Ediciones. 2005.

5. Pinilla AE. Construcción y evaluación de un perfil de competencias profesionales en medicina interna. Colección Desarrollo Humano. Bogotá: Editorial Universidad Nacional de Colombia; 2015.

6. Moncada LI, Pinilla AE. Investigación en educación. Rev Fac Med Univ Nac Colomb 2006; 54: 313-329.

7. Bonilla-Castro E, Rodríguez P. Más allá del dilema de los métodos. La investigación en ciencias sociales. Tercera Edición. Bogotá: Grupo Editorial Norma; 1995.

8. Kuhn, T.S. La estructura de las revoluciones científicas (primera reimpresión). Santafé de Bogotá, D.C.: Fondo de Cultura Económica; 1992.

9. Montaña C, Moreno C, Ramírez P. Pinilla AE, Serrano C. El conocimiento de las concepciones de los estudiantes universitarios: punto de partida para los lineamientos de una propuesta pedagógica. Tesis de maestría. Universidad Pedagógica Nacional; 1996.

10. García R. Las competencias de los alumnos universitarios. Revista Interuniversitaria de Formación del Profesorado 2006; 20 (3): 253-69.

11. Pinilla AE. Las competencias en la educación superior. En: Madiedo N, Pinilla AE, Sánchez J, editores. Reflexiones en educación universitaria II: Evaluación. Primera edición. Bogotá: El Malpensante S.A., 2002. p. 101- 35.

12. Denyer M, Furnemont J, Poulain R, Vanloubbeeck G. Las competencias en la educación. Un balance. (primera edición). México. D.F.: Fondo de Cultura Económica; 2007.

13. Pinilla AE, Páramo P. Fundamento de la postura epistemológica del maestro universitario-investigador. Entornos 2011; 24: 287-94. 